\title{
I-CONVERGENT SEQUENCE SPACES ASSOCIATED WITH MULTIPLIER SEQUENCES
}

\author{
BINOD CHANDRA TRIPATHY AND BIPAN HAZARIKA
}

Abstract. In this article we introduce the sequence spaces $c^{I}(\Lambda), c_{0}^{I}(\Lambda), m^{I}(\Lambda)$ and $m_{0}^{I}(\Lambda)$ associated with the multiplier sequence $\Lambda=\left(\lambda_{k}\right)$ of non-zero scalars. We study the different algebraic and topological properties of these sequence spaces like solidness, symmetricity, sequence algebra, convergence free etc. Also we characterize the multiplier problem and obtain some inclusion relation involving these sequence spaces.

Mathematics subject classification (2000): 40A05, 40C05.

Key words and phrases: Multiplier sequence spaces, Ideal, filter, $I$-convergent, $I$-Cauchy, solid space, symmetric space, sequence algebra, convergence free.

\section{REFERENCES}

[1] K. DemirCI, I-limit superior and limit inferior, Math. Commun., 6, (2001), 165-172.

[2] G. GOES, S. GOES, Sequences of bounded variation and sequences of Fourier coefficients, Math. Zeift., 118, (1970), 93-102.

[3] P. K. Kamthan, Bases in certain classes of Frechet spaces, Tamkang Jour. Math., (1976), 41-49.

[4] P. Kostyrko, T. ŠAlát, W. WiLCZYNSKI, I -convergence, Real Analysis Exchange, 26(2), (2000-2001), 669-686.

[5] P. Kostyrko, M. MaCAJ, T. ŠalÁt, Statistical convergence and I-convergence, Real Analysis Exchange (in Press).

[6] T. Šalát, B. C. TRIPATHY, M. Ziman, On some properties of I-convergence, Tatra Mt. Math. Publ., 28, (2004), 279-286.

[7] T. Šalát, B. C. TRipathy, M. Ziman, On I-convergence field, Italian Jour. Pure Appl. Math., 17, (2005), 45-54.

[8] B. C. TRIPATHY, Matrix transformations between some classes of sequences, J. Math. Anal. Appl., 206(2), (1997), 448-450.

[9] B. C. TRIPATHY, On some classes of difference paranormed sequence spaces associated with multiplier sequences, International Jour. Math. Sci., 2(1), (2003), 159-166.

[10] B. C. TRIPATHY, S. MAHANTA, On a class of vector valued sequences associated with multiplier sequences, Acta Math. Appl. Sinica., 20(3), (2004), 487-494.

[11] B. C. TRIPATHY, M. SEN, Vector valued paranormed bounded and null sequences associated with multiplier sequences, Soochow Jour. Math., 29(3), (2003), 313-325. 